\title{
The Tol1 transposable element of the medaka fish moves in human and mouse cells
}

\author{
Akihiko Koga $\cdot$ Atsuko Shimada \\ Toshiya Kuroki · Hiroshi Hori · Junko Kusumi · \\ Yoriko Kyono-Hamaguchi · Satoshi Hamaguchi
}

Received: 12 March 2007 / Accepted: 11 May 2007/Published online: 7 June 2007

(C) The Japan Society of Human Genetics and Springer 2007

\begin{abstract}
DNA-based transposable elements can be used as tools for gene engineering and gene therapy. A great advantage over RNA-mediated elements and retroviruses is the simplicity and safety of usage. The Toll element of the medaka fish Oryzias latipes has structural features of DNA-based elements. Although its excision has already been demonstrated, de novo insertion has not been observed, and a transposase has not been hitherto identified. We first cloned, through in silico search alignments and genomic library screenings, a 4.4-kb Toll copy carrying open reading frames and then identified, by mRNA analysis, a $2.9-\mathrm{kb}$ transcript coding for 851 amino acids. The protein product of this transcript catalyzed transposition of a nonautonomous Toll copy in human and mouse culture cells. This identification of a fully functional Toll transposase could lead to the development of new tools for
\end{abstract}

Electronic supplementary material The online version of this article (doi:10.1007/s10038-007-0161-2) contains supplementary material, which is available to authorized users.

A. Koga $(\bowtie) \cdot$ T. Kuroki $\cdot$ H. Hori

Division of Biological Science, Graduate School of Science,

Nagoya University, Nagoya 464-8602, Japan

e-mail: koga@bio.nagoya-u.ac.jp

A. Shimada

Department of Biological Sciences,

Graduate School of Sciences, University of Tokyo,

Tokyo 113-0033, Japan

J. Kusumi

Department of Biological Science, Graduate School of Science,

Kyushu University, Fukuoka 810-8560, Japan

Y. Kyono-Hamaguchi · S. Hamaguchi

Department of Environmental Science, Faculty of Science,

Niigata University, Niigata 950-2181, Japan basic and translational molecular biology applications in mammals.

Keywords Transposable element - Transposition . Genetic tools - Gene transfer vector

\section{Introduction}

Transposable elements can be divided into two main classes: RNA-mediated and DNA-based. The former include LINEs, SINEs, and retrovirus-like elements, and many such active elements have been identified in vertebrate genomes (Dombroski et al. 1991; Li et al. 2001; Burden et al. 2005). In contrast, active DNA-based elements are extremely rare in vertebrates, although defective elements are abundant (IHGSC 2001). The only DNAbased elements for which de novo transposition has been demonstrated are the Tol2 element of the medaka fish (Koga et al. 1996) and the Tzf element of zebrafish (Lam et al. 1996).

There is one other element for which part of the transposition reaction has been observed. The element is Toll, which resides as 100-200 copies in the genome of the medaka fish (Koga et al. 1995). It was first identified as an insertion sequence within the tyrosinase gene of a complete oculocutaneous albino mutant, tyrosinase being the key enzyme for biosynthesis of the black pigment melanin. Unlike the subsequently identified element Tol2, whose transposition activity was readily demonstrated soon after its discovery, Toll was initially thought to be extinct due to the absence of de novo excision or insertion. In addition, no gene-like structure could be identified in the Toll copies that were isolated, including the original element inserted in the tyrosinase gene (Koga et al. 1995). Recently, however, 
we identified a fish exhibiting mosaic melanin pigmentation in an albino substrain. We demonstrated by PCR analysis that excision of Toll occurs in somatic cells of fish from this substrain (Tsutsumi et al. 2006).

The occurrence of excision suggested the presence of an autonomous Toll copy with an intact and functional transposase gene in the mosaic fish. With this unique material, we proceeded to successfully identify and functionally validate the transposase gene and its protein product. The Toll element has now been shown to move in cultured human and mouse cells. The element can thus be expected to provide new genetic tools for the human and mouse and possibly also for other mammals.

\section{Materials and methods}

Fish

The original mutant medaka fish with a complete albino phenotype was first identified in a commercial breeding population more than 30 years ago (Tomita 1975), and a laboratory strain originating from this fish was established. The tyrosinase gene of this mutant carries a 1.9-kb copy of the Toll element inserted within its first exon (Koga et al. 1995). In 2001, an animal exhibiting mosaic melanin pigmentation was identified in a substrain maintained at Niigata University. Pigmentation was not observed in fish of the original strain kept at Nagoya University. The original albino strain and the melanin-exhibiting substrain were named $i^{1}$-Tomita and $i^{1}$-Niigata, respectively (Tsutsumi et al. 2006). In this paper, we have simplified their designations as substrains $\mathrm{A}$ and $\mathrm{B}$, respectively. Fish samples of these substrains used for the present study are also referred to as A and B, respectively. These substrains have not experienced outcrossing.

\section{Databases}

The following publicly available databases were used: the Medaka Genome Project (http://shigen.lab.nig.ac.jp/meda$\mathrm{ka} / \mathrm{genome} /$ ) for in silico assembly of a possible nucleotide sequence of a full-length Toll element; MOTIF (http:// motif.genome.jp/) for motif searching; Pfam (http:// www.sanger.ac.uk/Software/Pfam/) for collecting sequences of $h A T$ family transposases; and Clustal X (http:// bips.u-strasbg.fr/fr/Documentation/ClustalX/) for amino acid sequence alignments.

Reagents and kits

The following molecular biology reagents and kits were utilized according to manufacturer instructions: PCR enzyme ExTaq (Takara Bio, Otsu, Japan) for PCR amplification; fosmid vector pCC1FOS (EPICENTRE Biotechnologies, Madison, WI, USA) for construction of genomic libraries; AlkPhos Direct Labelling and Hybridization System (GE Healthcare, Chalfont St. Giles, UK) for probe labeling and hybridization analyses; RNeasy kit (QIAGEN, Hilden, Germany) for RNA extraction; FirstChoice RLMRACE Kit (Ambion, Austin, TX, USA) for RACE analyses; PolyFect Transfection Reagent (QIAGEN) for transfection of cells; G418 (Invitrogen, Carlsbad, USA) for G418 selection. Specific experimental conditions are described elsewhere in the text and figure legends.

Transposition assays with mammalian cell cultures

Human HeLa and mouse NIH/3T3 cells were maintained in DMEM with $10 \%$ FBS and antibiotics in an incubator at $37^{\circ} \mathrm{C}$ with $5.0 \% \mathrm{CO}_{2}$.

Aliquots of $1 \times 10^{5}$ cells were seeded in $35-\mathrm{mm}$ dishes and incubated for $24 \mathrm{~h}$. Mixtures of plasmid DNAs, $1,000 \mathrm{ng}$ for each dish, were then introduced into the cells using the PolyFect reagent. After a further $24 \mathrm{~h}$ incubation, cells were washed twice with PBS and incubated for another $24 \mathrm{~h}$ in fresh medium without plasmid DNA and transfection reagent. Cells were then trypsinized, suspended in $2.0 \mathrm{ml}$ of medium, and $100 \mu \mathrm{l}$ aliquots of cell suspension were transferred to dishes of different sizes (35, 60 and $90 \mathrm{~mm}$ ) containing medium and $500 \mu \mathrm{g} / \mathrm{ml} \mathrm{G} 418$. After 12 days under G418 selection, cells were fixed with $20 \%$ formalin and stained with Giemsa's solution. Cell colonies were counted in dishes containing colony numbers closest to 100 , and colony numbers per $10^{5}$ seeded cells were determined. The transfection assays were carried out in triplicate.

\section{Results}

Fish material was improved

The frequency of individuals with mosaic pigmentation in fish of substrain B was approximately $20 \%$ just after the detection in 2001. To enhance molecular analyses, we successively selected fish with denser pigmentation for single-pair matings over five generations. The frequency thus became more than $90 \%$ and the pigmented area also became larger (Fig. 1).

A putative full-length Toll element was identified through in silico assembly

The original Toll copy isolated from the tyrosinase gene (Toll-tyr) is $1.9 \mathrm{~kb}$ in length. Because internal deletion is a common mechanism for generating nonautonomous copies 
Fig. 1a-c Fish exhibiting mosaic melanin pigmentation. a Fish with broad pigmentation on eyes and many pigmented spots on the dorsal skin. b Fish exhibiting spoke-like pigmentation in the eyes. c Peritoneum, which is densely pigmented in wild-type fish and unpigmented in albino fish
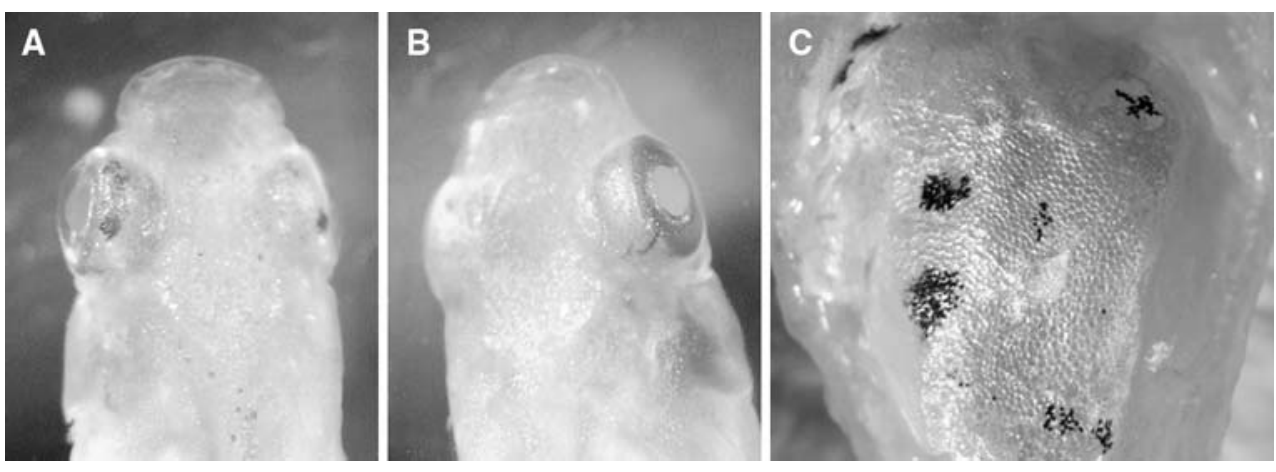

of DNA-based transposable elements (O'Hare and Rubin 1983), we conducted repeated database searches for longer copies, using the Toll-tyr sequence (DDBJ D42062) for initial query. In each round, when a new sequence consisting of the query sequence and an additional segment was present more than once in the database, we assumed that it belonged to the full-length Toll sequence and used the additional portion as a query sequence for the next round of database search. In this way, information on partly overlapping segments accumulated. We then merged the sequences, obtaining a consecutive sequence of $4.4 \mathrm{~kb}$. A new database search using the entire $4.4-\mathrm{kb}$ sequence as query was conducted to refine this sequence by adopting the consensus nucleotide at every position. This search provided a sequence containing some open reading frames (ORFs) of up to $2.3 \mathrm{~kb}$ within the $4.4-\mathrm{kb}$ Toll sequence, which was designated Toll-L0. This hypothetical, assembled sequence contained several surplus stop codons as compared with the actual sequence of the cloned fragment Tol1-L1 described below. The entire 4.4-kb region still does not appear as a nondisrupted sequence in the medaka fish sequence database (version 43 released in February 2007).

\section{Full-length Toll elements were cloned}

PCR amplification was performed on genomic DNA from the mosaic pigmentation medaka substrain (referred to as fish B) to amplify a 1.2-kb fragment of the Toll-L0 sequence (Fig. 2, segment b). Using this amplified fragment as a probe, we screened a genomic library of fish B by the colony hybridization method. Three clones having different flanking sequences, Toll-L1 to Toll-L3, were isolated, and sequenced for their entire Toll regions. Toll-L1 and TollL3 exhibited an identical sequence of 4,355 bp. Tol1-L2 is $4,354 \mathrm{bp}$ in length and the only difference is in the number of consecutive $\mathrm{G}$ residues located close to the $3^{\prime}$ end (21 G's in Tol1-L1 and Toll-L3; $20 \mathrm{G}^{\prime}$ s in Tol1-L2). The sequence of Tol1-L1 has been deposited in DDBJ under the accession number AB288091. Its structure, including information obtained from further analyses explained below, is shown in Fig. 2, together with its structural relationship to Toll-tyr.

The sequences of Toll-L1 to Toll-L3 contain the entire sequence of a subsequently identified transposase cDNA, except for the poly(A) tail region, with no mismatching. The difference in the number of consecutive $\mathrm{G}^{\prime} \mathrm{s}$ is downstream of the transcribed region. Another fact explained below is that fish B exhibited three hybridization bands in a genomic Southern blot analysis using segment $b$ as a probe. Therefore, one or more of these three Toll elements are the source of transposase RNA.

A BLAST search using the sequences of genomic regions flanking the Tol1-L1 to Tol1-L3 elements as queries indicated that Toll-L1 and Toll-L2 are located on chromosome 21 and Toll-L3 on chromosome 16 (data not shown). A Toll element is not present at these positions in the fish used as material for the database (strain Hd-rR).

\section{A cDNA encoding a putative Toll transposase} was identified

We performed 3' RACE (rapid amplification of cDNA ends) of the putative Toll transposase transcript using RNA extracted from the original albino strain (fish A) and the mosaic pigmentation substrain (fish B). Southern blot analysis of 3' RACE products resulted in a positive hybridization signal from fish $\mathrm{B}$ but not from fish $\mathrm{A}$ (Fig. 3), indicating the presence of a Toll transcript from the ORF in fish B and the absence, or presence of only very small amounts, in fish A. Subsequently, 5' RACE analysis of fish B also yielded a single hybridizing band (Fig. 3). Further cloning and sequencing of the RACE fragments responsible for the signals resulted in a $2.9-\mathrm{kb}$ cDNA sequence containing a single ORF. We have deposited this sequence in DDBJ under the accession number AB264112. By alignment of this full-length cDNA to the Toll-L1 element, we determined the Toll transposase gene to comprise three exons (Fig. 2).

A BLAST search of protein databases using the predicted amino acid sequence of the Toll ORF produced a 


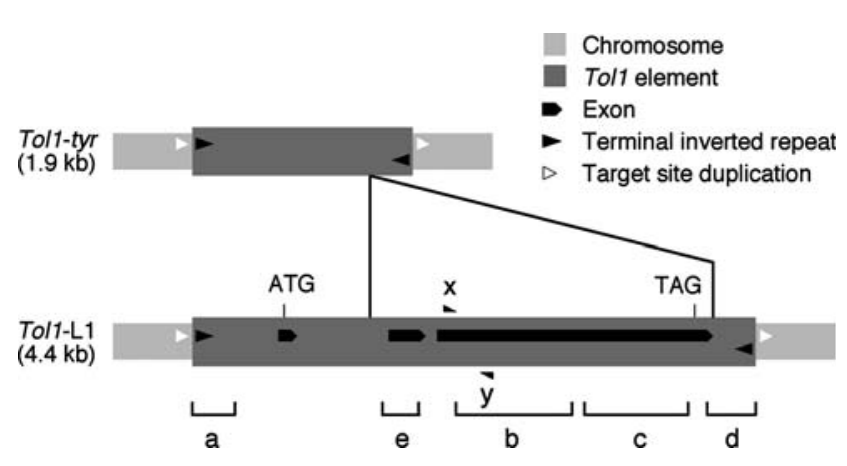

Fig. 2 Structure of nonautonomous and autonomous copies of Toll. Toll-tyr is the original, nonautonomous copy of Toll that was inserted within the tyrosinase gene of fish A. Toll-L1 is the complete, autonomous copy carrying a fully functional transposase gene identified in the present study. It was identified by screening a genomic library of fish $\mathrm{B}$, constructed by inserting $36-48 \mathrm{~kb}$ of mechanically sheared genomic fragments into the fosmid vector pCC1FOS. The number of colonies screened was $1.2 \times 10^{5}$, which is equivalent to a more than $2 \times$ coverage of the medaka haploid genome. The transposase gene is indicated in black, and positions of the start codon $(A T G)$ and stop codon $(T A G)$ are shown. Bracketed segments $a-e$ indicate various DNA fragments used to generate hybridization probes, and $x$ and $y$ refer to the positions of the forward and reverse PCR primers used in the $3^{\prime}$ and $5^{\prime}$ RACE experiments, respectively. Primers $x$ and $y$ are nt $152-181$ and nt 357-332 of AB264112, respectively

list of similarity hits consisting mainly of $h A T$ family transposable elements (data not shown). The $h A T$ family includes hobo of Drosophila, Activator of maize and Tam3 of snapdragon, and their members are distributed in a wide range of organisms, even across kingdoms (Calvi et al. 1991). Tol2 of the medaka fish is also a member of this family (Koga et al. 1999, 2006).

Most Toll copies in the medaka fish genome are defective

Our initial database search suggested that internal regions of Toll are less frequent in the medaka fish genome than terminal regions. This was confirmed by Southern blot analysis of several medaka fish strains using probes representing different portions of the full-length Toll-L1 element. Hybridization with a probe to the central region produced zero to five hybridization bands, while terminal region probes detected more than 100 bands each (Fig. 4). Such a phenomenon is common among DNA-based transposable elements, including the maize Activator element (Rubin and Levy 1997) and the Drosophila $P$ element (O'Hare and Rubin 1983). A widely accepted explanation is that internal deletion is the main mechanism for generation of nonautonomous copies from autonomous elements (O'Hare and Rubin 1983). This also appears to be true for Toll.

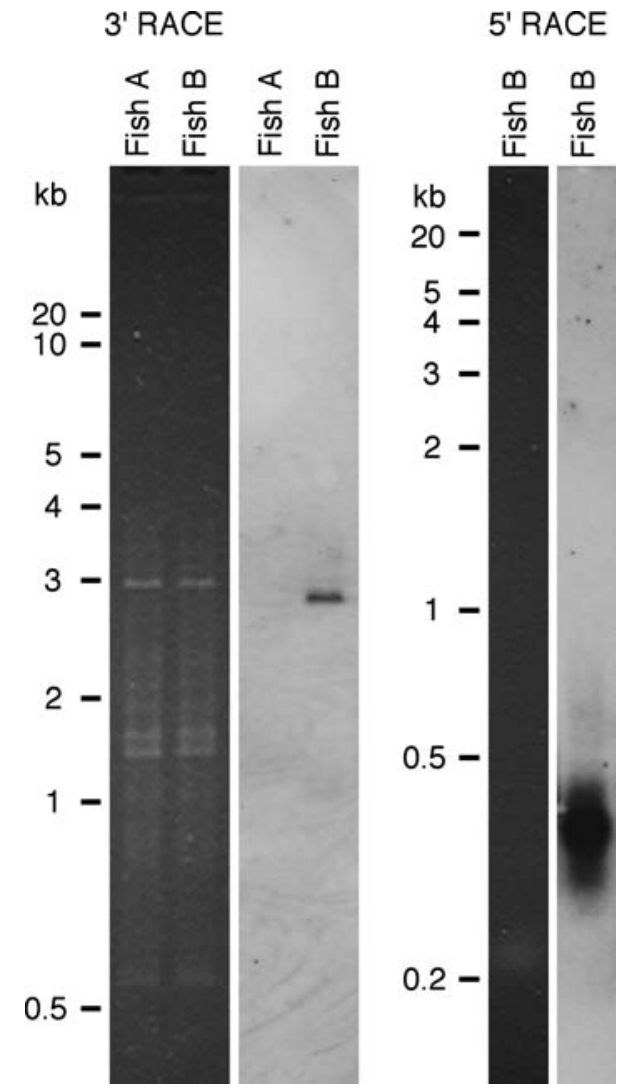

Fig. 3 Results of RACE analyses. Total RNA was extracted from six embryos of fish A and fish B at 7 days post-fertilization, and firststrand cDNAs were synthesized with an oligo-dT primer. $3^{\prime}$ RACE of first-strand cDNA was performed using primer $x$ and a $3^{\prime}$ adaptor primer included in the RACE kit. PCR products were electrophoresed on a $1.0 \%$ agarose gel, transferred to a nylon membrane and hybridized with probe $\mathrm{b}$. The left panel shows the results after agarose gel electrophoresis, and the right panel shows the autoradiograph after probe hybridization. A hybridizing band was observed only from fish B. Subsequently, a gel piece corresponding to the signal was isolated, and recovered DNA fragments were ligated to a plasmid vector, and a clone hybridizing with probe $\mathrm{b}$ was selected by the colony hybridization method. $5^{\prime}$ RACE analysis was performed only on the RNA from fish $\mathrm{B}$, using primer $y$ and a $5^{\prime}$ adaptor primer included in the kit. PCR products were processed as in the $3^{\prime}$ experiment, except that hynridization probe e was used. A hybridizing band was observed, and its corresponding fragment was similarly cloned

Toll is transposed in mammalian cells

To test whether the Toll ORF encodes a functional transposase capable of mediating transposition of the Toll element, we first constructed a donor plasmid carrying the 1.9-kb Toll-tyr element containing an embedded neomycin-resistance gene, and a helper plasmid containing the Toll ORF driven by the CMV promoter and stabilized by a polyadenylation signal (Fig. 5a). As negative controls, we also prepared a defective helper plasmid in which two internal codons of the ORF were modified into stop codons, 
Fig. 4 Southern blot analysis of Toll copies in medaka fish genome. Genomic DNA was extracted from fish A, fish B, and fishes from two other highly inbred laboratory strains, HNI and Hd-rR, which are commonly used in medaka research. For each fish, $8.0 \mu \mathrm{g}$ of genomic DNA was digested to completion with the restriction enzyme $P v u \mathrm{II}$, electrophoresed on a $1.0 \%$ agarose gel, transferred to a nylon membrane and then hybridized with probes a-d (see Fig. 2 for locations). On the left margin are indicated the positions of DNA fragments used as size markers. The autoradiography results indicate that the majority of Toll copies in the medaka fish genome contain internal deletions



as well as a "filler" plasmid carrying an unrelated DNA fragment of the same size as the Toll ORF (Fig. 5a). The latter plasmid was used in subsequent gene transfer assays to maintain equivalent DNA intake efficiency with constant amounts of total DNA. Donor, helper, and/or control plasmid DNAs were introduced into human HeLa and mouse NIH/3T3 cells, and transformants with G418-resistance were selected. Cells co-transfected with donor and helper plasmids produced more numerous G418-resistant colonies as compared with cells in which the helper plasmid was replaced with defective helper and/or filler plasmid (Fig. 5b).

To confirm that the transformations had been caused by transposition of Toll into the host genomes, we cloned Toll-containing chromosomal DNA fragments from G418resistant cells after co-transfection with donor and helper plasmids and sequenced the Toll termini and their flanking regions. All eight clones analyzed were found to carry flanking sequences different from those of the donor plasmid and from each other. Eight base-pair target site duplications were also detected in all eight clones, indicating that the Toll portion of the donor plasmid was integrated into the chromosomes by transposition (see Supplementary material). The same analysis was performed with G418resistant cells after co-transfection with donor and defective helper plasmids. Two clones were sequenced, and the sequences of Toll-flanking regions were identical to those of the donor plasmid. These results demonstrate that the Toll ORF encodes a functional Toll transposase enzyme.

Transposition frequency is correlated with amount of transposase

To determine the relationship between the amount of the transposase and the transposition frequency, we measured the frequency in HeLa cells by performing co-transfections of a fixed amount of the donor plasmid and varying amounts of the helper plasmid. The ratio of donor to helper plasmid ranged between 1:0.5 and 1:9. Within this range, the transposition frequency was positively correlated with increasing ratios (Fig. 5c).

\section{Discussion}

Identification of a functional transposase

Although a Toll element was identified earlier than Tol2, its de novo transposition could not then be demonstrated, presumably due to internal mutations and deletions in this particular Toll copy and most, or possibly all, of the other copies in the genome. We have now demonstrated the existence of a 

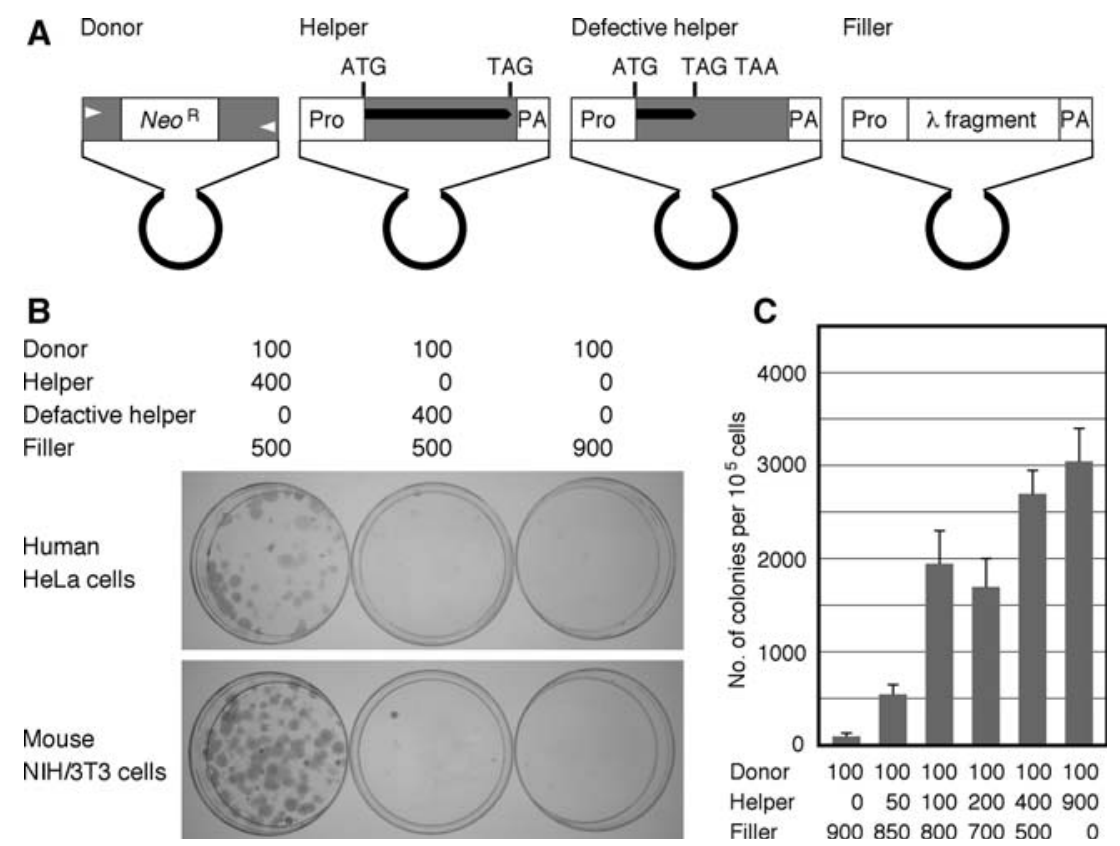

Fig. 5a-c Transposition assays. a Plasmids used for assays. The Toll-tyr element (DDBJ D42062), together with its flanking 8-bp TSDs (sequence CCTTTAGC), was amplified by PCR from genomic DNA of fish A, and cloned into plasmid pUC19. Subsequently, a segment of plasmid pCMV-Tag1 (nt 1,675-3,474 of DDBJ AF025668) including the neomycin-resistance gene was amplified by PCR and inserted into a single SalI recognition site present in Toll-tyr (nt 706-711 of D42062). The resultant construct was used as a donor plasmid. A helper plasmid was constructed by inserting the coding region of the Toll cDNA (nt 31-2,817 of AB264112) into the multiple cloning site of plasmid $\mathrm{pCI}$, in between the CMV promoter and the polyadenylation signal. A defective helper plasmid was created by introducing nucleotide alterations by PCR. The sequence for nt 996-1,001 is ATGAAA, which corresponds to amino acids methionine and lysine in the transposase. This sequence was changed to TAGTAA, which resulted in the introduction of two consecutive

naturally occurring, complete Toll element and a transposase capable of the entire reaction of transposition.

Possible cause of resumption of transposition

We examined the body color phenotypes of more than 2,000 fish of substrain A for melanin pigmentation, but no pigmented fish could be detected. It is a widely accepted idea from the viewpoint of evolution that natural selection acts against high activity of transposable elements because of their deleterious effects on their host organisms (Hartl et al. 1997). Toll elements of substrain A appear to have been stabilized as a result of natural selection, like most of the transposable elements found in various organisms. In contrast to substrain A, more than $90 \%$ of fish of substrain $\mathrm{B}$ exhibit mosaic pigmentation. These observations raise a question that needs to be solved: what caused activation of Toll only in substrain B? stop codons in the middle of the transposase ORF. A filler plasmid was constructed by inserting a $\lambda$ DNA fragment of $2.8 \mathrm{~kb}$ into pCI in place of Toll transposase cDNA. b Examples of surviving colonies. $\mathrm{HeLa}$ and NIH/3T3 cells were co-transfected with donor plasmid together with either helper or defective helper plasmids and/or filler plasmids in various amounts (nanograms) and then selected with G418. Photos of 60-mm dishes stained with Giemsa's solution are shown. Only cells co-transfected with donor and helper plasmid produced significant numbers of G418-resistant colonies. c Transposition frequency of Toll in HeLa cells. Transposition assays were conducted using various amounts of the helper plasmid. Each assay was conducted using a total of $1,000 \mathrm{ng}$ of plasmid DNA. The amounts of the donor, helper and filler plasmids are indicated under each bar. The heights of the bars indicate the mean colony numbers from three independent assays, with standard errors of the mean indicated by the vertical lines

Both substrains are descendants of a common albino strain and have been maintained without crossing to other fish strains or even to each other, indicating that a fulllength Toll copy had already been present in the genome of the original albino strain or that the element was synthesized within substrain B by some mechanism such as DNA recombination or base substitution. If the former is the case, it is more likely that substrain A also contains a potentially autonomous Toll element than the explanation that such an element was lost in substrain A, because there are many other substrains that do not show any sign of melanin pigmentation (personal communications from the medaka research community). Our results of Southern blot analysis (Fig. 4) indicate that both substrains possess DNA fragments hybridizing to the central region of Toll-L1 (probe b), which is not inconsistent with the explanation that substrain A contains a potentially autonomous Toll element. It should be noted that the sizes of hybridization 
bands differ between the two substrains. The restriction enzyme used for our Southern blot analysis is $P v u \mathrm{II}$, whose recognition site appears only once in the Toll-L1 sequence. Therefore, differences in the band size between the two substrains could be due to differences in the chromosomal positions of causative Toll copies. One possible explanation would be that Toll elements move even in substrain A, at an extremely low frequency, and that a transposition event of a potentially autonomous element to a specific chromosomal position resulted in a high-level expression of the transposase gene in substrain B. The currently active Toll copy in substrain B may have acquired activity by falling into a DNA region that is under the effect of a strong promoter of a neighboring host gene. This idea is consistent with the observation that the transposase gene does not have an apparent promoter-like sequence in its upstream region within the Toll-L1 sequence.

\section{Possibility of developing genetic tools}

DNA-based transposable elements can be used as tools for gene transfer, mutagenesis, gene/promoter/enhancer trapping, and gene therapy. A great advantage over RNAmediated elements and retroviruses is the simplicity and safety of usage. The identification of a functional Toll transposase could lead to the development of new tools for such applications. The naturally occurring Tol2 element has already been applied for such purposes (Koga et al. 2002; Johnson Hamlet et al. 2006; Choo et al. 2006), together with the molecularly resurrected Sleeping Beauty (Ivics et al. 1997) and Frog Prince (Miskey et al. 2003) elements from the salmonid and frog genomes, respectively, and the piggyBAC element originating from an insect (Wu et al. 2006).

While Toll and Tol2 are both members of the hAT family, the molecularly reconstructed elements Sleeping Beauty and Frog Prince belong to the mariner/Tcl family. The piggyBAC element is of another type. One major difference between these transposable element groups is their element size: most elements of the mariner/Tc 1 family are $1-2 \mathrm{~kb}$ and piggyBAC is $2.5 \mathrm{~kb}$ in size, while "fulllength" elements of the $h A T$ family are typically $4-6 \mathrm{~kb}$ long. Because a negative correlation between element size and transposition frequency is often observed (Geurts et al. 2003), the larger size of the $h A T$ family elements might be an advantage for carrying large DNA fragments. Actually, we have previously shown that a Tol2 element as large as $9.0 \mathrm{~kb}$ can be transposed (Koga et al. 2002). Another important difference between the transposable element groups involves the tendency for "overexpression inhibition." Excess transposase is known to decrease the transposition frequency of Sleeping Beauty (Geurts et al. 2003) and piggyBAC (Wu et al. 2006). However, such a phenomenon was not observed with Toll in the present study (Fig. 5c) or in another experiment with Tol2 (Kawakami and Noda 2004).

The Toll and Tol2 transposable element systems appear to be independent of each other because we have not observed transposition of Toll in medaka fish strains in which Tol2 is highly active and vice versa. The availability of two transposable element systems of the $h A T$ family can be expected to expand technology for genetic engineering in mammals. One possible application would be to send sequentially two different genes, such as mutually complementary genes or mutually antagonistic genes, into a single cell line or animal. Subsequent removal of one of the two genes may be possible by inducing excision of one element.

Database searches have indicated that the nucleotide sequence similarity is higher in terminal regions than internal regions of Toll, suggesting that only terminal regions are sufficient for transposition reactions provided that the transposase is supplied. Experiments to determine the minimum necessary sequences are now in progress.

Acknowledgements We are grateful to Akira Tachibana, Hidenori Tachida, Malcolm Moore and Samuel S. Chong for helpful discussion. Human HeLa cells and mouse NIH/3T3 cells were obtained from the HSRRB of the Japan Health Sciences Foundation (registry no. JCRB9004 and JCRB0615, respectively). This work was supported by grant no. 16570002 to A.K. from the Ministry of Education, Culture, Sports, Science and Technology of Japan and a Basic Science Research Grant from the Sumitomo Foundation to A.K.

\section{References}

Burden AF, Manley NC, Clark AD, Gartler SM, Laird CD, Hansen RS (2005) Hemimethylation and non-CpG methylation levels in a promoter region of human LINE-1 (L1) repeated elements. J Biol Chem 280:14413-14419

Calvi BR, Hong TJ, Findley SD, Gelbart WM (1991) Evidence for a common evolutionary origin of inverted repeat transposons in Drosophila and plants: hobo, Activator, and Tam3. Cell 66:465471

Choo BG, Kondrichin I, Parinov S, Emelyanov A, Go W, Toh WC, Korzh V (2006) Zebrafish transgenic Enhancer TRAP line database (ZETRAP). BMC Dev Biol 6:5

Dombroski BA, Mathias SL, Nanthakumar E, Scott AF, Kazazian HH $\mathrm{Jr}$ (1991) Isolation of an active human transposable element. Science 254:1805-1808

Geurts AM, Yang Y, Clark KJ, Liu G, Cui Z, Dupuy AJ, Bell JB, Largaespada DA, Hackett PB (2003) Gene transfer into genomes of human cells by the Sleeping Beauty transposon system. Mol Ther 8:108-117

Hartl DL, Lozovskaya ER, Nurminsky DI, Lohe AR (1997) What restricts the activity of mariner-like transposable elements. Trends Genet 13:197-201

International Human Genome Sequencing Consortium (2001) Initial sequencing and analysis of the human genome. Nature 409:860921 
Ivics Z, Hackett PB, Plasterk RH, Izsvak Z (1997) Molecular reconstruction of Sleeping Beauty, a Tc1-like transposon from fish, and its transposition in human cells. Cell 91:501-510

Johnson Hamlet MR, Yergeau DA, Kuliyev E, Takeda M, Taira M, Kawakami K, Mead PE (2006) Tol2 transposon-mediated transgenesis in Xenopus tropicalis. Genesis 44:438-445

Kawakami K, Noda T (2004) Transposition of the Tol2 element, an Ac-like element from the Japanese medaka fish Oryzias latipes, in mouse embryonic stem cells. Genetics 166:895-899

Koga A, Inagaki H, Bessho Y, Hori H (1995) Insertion of a novel transposable element in the tyrosinase gene is responsible for an albino mutation in the medaka fish, Oryzias latipes. Mol Gen Genet 249:400-405

Koga A, Suzuki M, Inagaki H, Bessho Y, Hori H (1996) Transposable element in fish. Nature 383:30

Koga A, Suzuki M, Maruyama Y, Tsutsumi M, Hori H (1999) Amino acid sequence of a putative transposase protein of the medaka fish transposable element Tol2 deduced from mRNA nucleotide sequences. FEBS Lett 461:295-298

Koga A, Hori H, Sakaizumi M (2002) Gene transfer and cloning of flanking chromosomal regions using the medaka fish Tol2 transposable element. Mar Biotechnol 4:6-11

Koga A, Iida A, Hori H, Shimada A, Shima A (2006) Vertebrate DNA transposon as a natural mutator: the medaka fish Tol2 element contributes to genetic variation without recognizable traces. Mol Biol Evol 23:1414-1419

Lam WL, Lee TS, Gilbert W (1996) Active transposition in zebrafish. Proc Natl Acad Sci USA 93:10870-10875
Li X, Scaringel WA, Hill KA, Roberts S, Mengos A, Careri D, Pinto MT, Kasper CK, Sommer SS (2001) Frequency of recent retrotransposition events in the human factor IX gene. Hum Mutat 17:511-519

Miskey C, Izsvak Z, Plasterk RH, Ivics Z (2003) The Frog Prince: a reconstructed transposon from Rana pipiens with high transpositional activity in vertebrate cells. Nucleic Acids Res 31:68736881

O'Hare K, Rubin GM (1983) Structures of P transposable elements and their sites of insertion and excision in the Drosophila melanogaster genome. Cell 34:25-35

Rubin E, Levy AA (1997) Abortive gap repair: underlying mechanism for Ds element formation. Mol Cell Biol 17:6294-6302

Tomita H (1975) Mutant genes in the medaka. In: Yamamoto T (ed) Medaka (killifish): biology and strains. Yugakusha, Tokyo, pp 251-272

Tsutsumi M, Imai S, Kyono-Hamaguchi Y, Hamaguchi S, Koga A, Hori $\mathrm{H}$ (2006) Color reversion of the albino medaka fish associated with spontaneous somatic excision of the Tol-1 transposable element from the tyrosinase gene. Pigment Cell Res 19:243-247

Wu SC, Meir YJ, Coates CJ, Handler AM, Pelczar P, Moisyadi S, Kaminski JM (2006) piggyBac is a flexible and highly active transposon as compared to Sleeping Beauty, Tol2, and Mos1 in mammalian cells. Proc Natl Acad Sci USA 103:1500815013 